\title{
Stretch Evoked Potentials in Healthy Subjects and After Stroke: A Potential Measure for Proprioceptive Sensorimotor Function
}

\author{
Sanne Floor Campfens, Carel G. M. Meskers, Alfred C. Schouten, Michel J. A. M. van Putten, and \\ Herman van der Kooij
}

\begin{abstract}
Sensory feedback is of vital importance in motor control, yet rarely assessed in diseases with impaired motor function like stroke. Muscle stretch evoked potentials (StrEPs) may serve as a measure of cortical sensorimotor activation in response to proprioceptive input. The aim of this study is: 1) to determine early and late features of the StrEP and 2) to explore whether StrEP waveform and features can be measured after stroke.

Consistency of StrEP waveforms and features was evaluated in 22 normal subjects. StrEP features and similarity between hemispheres were evaluated in eight subacute stroke subjects.

StrEPs of normal subjects had a consistent shape across conditions and sessions (mean cross correlation waveforms $>0.75$ ). Stroke subjects showed heterogeneous StrEP waveforms. Stroke subjects presented a normal early peak $(40 \mathrm{~ms}$ after movement onset) but later peaks had abnormal amplitudes and latencies. No significant differences between stroke subjects with good and poor motor function were found $(p>0.14)$.

With the consistent responses of normal subjects the StrEP meets a prerequisite for potential clinical value. Recording of StrEPs is feasible even in subacute stroke survivors with poor motor function. How StrEP features relate to clinical phenotypes and recovery needs further investigation.
\end{abstract}

Index Terms-Electroencephalography (EEG), evoked potentials, muscle stretch, proprioception, stroke.

\section{INTRODUCTION}

$\mathbf{S}$ TROKE is a leading cause of adult-onset disability in the western world. Rehabilitation after stroke aims at reducing motor impairment via restitution - actual return of motor func-

Manuscript received December 20, 2013; revised May 23, 2014, November 08, 2014; accepted November 30, 2014. Date of publication January 07, 2015; date of current version July 03, 2015. The work of C. G. M. Meskers was supported by a fellowship (F2011(1)-25) from the Hersenstichting, The Netherlands.

S. F. Campfens is with the Laboratory of Biomechanical Engineering and also the Clinical Neurophysiology Chair, MIRA Institute of Biomedical Engineering and Technical Medicine, University of Twente, Twente, The Netherlands (e-mail: s.f.campfens@alumnus.utwente.nl).

C. G. M. Meskers is with the Department of Rehabilitation Medicine, Leiden University Medical Center, Leiden, The Netherlands, and also with the VU University Medical Center, Amsterdam, the Netherlands.

A. C. Schouten and H. van der Kooij are with the Laboratory of Biomechanical Engineering, MIRA Institute of Biomedical Engineering and Technical Medicine, University of Twente, The Netherlands, and also with the Department of Biomechanical Engineering, Delft University of Technology, Delft, The Netherlands.

M. J. A. M van Putten is with the Clinical Neurophysiology Chair, MIRA Institute of Biomedical Engineering and Technical Medicine, University of Twente, Twente, The Netherlands, and also with the Department of Neurology and Clinical Neurophysiology, Medisch Spectrum Twente, The Netherlands.

Color versions of one or more of the figures in this paper are available online at http://ieeexplore.iee.org.

Digital Object Identifier 10.1109/TNSRE.2015.2388692 tion - and compensation - the emergence of new movement patterns [1]. Although proprioception, stereognosis (i.e., the ability to recognize objects without visual input) and tactile sensation are commonly impaired after stroke [2], assessment and training of sensory modalities is generally not part of regular therapy. This is surprising given that voluntary motor control takes place in a closed loop where sensory feedback is essential [3]. Indeed, there is evidence that training of sensory function reduces impairment after stroke [4].

Integrity of the sensory pathways can be assessed by measuring the cortical response, by means of electroencephalography (EEG) or magnetoencephalography (MEG), to an external stimulus. The electrically elicited somatosensory evoked potential (SSEP) is used in clinical practice to evaluate whether sensory input is processed by the brain, for example in comatose patients or patients unable to communicate [5]. The early peaks of an evoked potential (within the first 30 or $40 \mathrm{~ms}$ after the stimulus in case of the median nerve SSEP) represent the arrival of sensory information at the cortex, indicating the integrity of the afferent sensory pathways. Later peaks are associated with processing of the sensory input [6], [7].

Abnormal SSEP waveforms were found in patients after stroke, indicating the presence of sensory deficits [8]. Presence, latency and amplitude of the early peaks of the SSEP (N20 and N20-P25) were found to normalize during motor recovery after stroke [9], [10]. Some studies even found the early peaks of the SSEP to be of predictive value regarding motor recovery after stroke; absence of the SSEP early after stroke is associated with poor motor recovery [11], [12].

Electrical stimulation of the median nerve provides a mixed artificial activation of sensory fibres, mainly related to tactile sensation [13], which does not necessarily reflect sensory feedback relevant for motor control. Real joint movement and muscle stretch also activate sensory fibers. This elicits a cortical response which can resemble sensory feedback and subsequent sensorimotor integration in movement control more closely, with a large contribution of muscle spindle afferent feedback [14]-[18]. It was shown that such a muscle stretch evoked potential (StrEP) has a different shape and involves activation of other cortical areas compared to the electrical SSEP [16], [17]. More specifically, the sources generating the first peaks of the StrEP are positioned a few centimeters anterior of the sources of the first peak (N20) of the electrical SSEP. Authors concluded that the StrEP is generated, at least in part, by the primary motor cortex [16], [17], while the electrical SSEP is generated by the primary sensory cortex [13]. 
Prevalence of proprioceptive deficits was found to exceed that of tactile deficits after stroke [2]. Muscle stretch addresses the afferent volley involved in motor control, mainly proprioception, and activates cortical motor areas. Muscle stretch may therefore be an attractive way of assessing integration of sensory feedback in motor function during motor recovery after stroke. As most of the motor function recovery happens within the first weeks after stroke [1], assessing proprioceptive function objectively in the subacute phase can provide additional insight in the role of sensory feedback in the recovery of motor control. However, before the potential clinical value of the StrEP can be established, invariant features of the StrEP need to be determined in a normal population. Furthermore, the feasibility of estimating StrEPs in the subacute phase after stroke should be demonstrated.

The aim of the current study is two-fold: first, we identify early and late components of the StrEP and their variability across stretch amplitudes and measurement sessions in a group of normal subjects. Consistent presence of the StrEP in normal subjects is a prerequisite for potential clinical value of the StrEP in patients; when the StrEP cannot be elicited consistently in normal subjects, absence or alteration of the StrEP cannot be related to pathology. Secondly, we explore StrEPs in a small group of subacute stroke survivors to evaluate whether the StrEPs and StrEP components can be measured in stroke survivors in the acute phase.

\section{MethodS}

\section{A. Subjects}

Twenty-two healthy volunteers (nine women, mean age 27 years, age range $23-35$, four subjects were left-hand dominant) participated in this study as normal subjects. The dominant hand was determined using the Dutch handedness questionnaire [19].

Eight first-ever hemispheric stroke patients participated in this study (one woman, mean age 57 years, age range 35-77). All stroke subjects were measured within 6 months post stroke (range 13-135 days post stroke). Stroke subject details are presented in Table I. Lesion location and class were determined based on clinical signs and CT images. In the Netherlands, the use of MRI imaging is not part of standard clinical care for this patient group. All stroke subjects had normal wrist proprioceptive function according to the Erasmus MC Modifications of the (revised) Nottingham Sensory Assessment [20]. Within the group of stroke subjects a distinction was made between subjects with good and poor motor function based on the Brunström Fugl-Meyer (FM) upper extremity score [21]. Five stroke subjects had a FM-UE of 63 or higher (maximum FM-UE score is 66). These subjects were able to move outside synergistic movement patterns, but some subjects experienced some loss of muscle strength. These five stroke subjects were considered to have good motor function. The remaining three subjects had considerably lower FM-UE scores and scored less than 30 points. These subjects were unable to move outside synergistic movement patterns and experienced severe loss of muscle strength and coordination. These three subjects were considered to have poor motor function.

All measurements were conducted in accordance with the Declaration of Helsinki. The measurements with the normal subjects were performed at the University of Twente and
TABLE I

Overview of Stroke SubJects. Lesion WAs Determined BASEd on CT-Imaging and Clinical Signs. MCA: Middle Cerebral Artery. Motor Function Is EVAluATEd USING BRUNNSTRÖM FugL-MEYER (FM) TEST FOR UPPER EXTREMITY (FM-UE, MAXIMAL SCORE: 66) [21]. Spasticity at Wrist Is Assessed Using Modified AshWorth ScAle (AS) [26]. ACT.: ACTIVE TASK, REL.: RELAXED TASK

\begin{tabular}{lcccccc}
\hline code & age & lesion & $\begin{array}{c}\text { days since } \\
\text { lesion }\end{array}$ & $\begin{array}{c}\text { FM- } \\
\text { UE }\end{array}$ & $\begin{array}{c}\text { AS } \\
\text { wrist }\end{array}$ & task \\
\hline P01 & 77 & $\begin{array}{c}\text { MCA-1, } \\
\text { subcortical }\end{array}$ & 106 & 65 & 0 & act. \\
\hline P02 & 58 & $\begin{array}{c}\text { MCA-r, } \\
\text { subcortical }\end{array}$ & 36 & 63 & 0 & act. \\
\hline P03 & 59 & $\begin{array}{c}\text { MCA-r, } \\
\text { subcortical }\end{array}$ & 135 & 26 & $\begin{array}{c}1+ \\
\text { (flex) }\end{array}$ & rel. \\
\hline P04 & 58 & $\begin{array}{c}\text { MCA-r, } \\
\text { cortical }\end{array}$ & 81 & 28 & $\begin{array}{c}1+ \\
\text { (flex) }\end{array}$ & rel. \\
\hline P05 & 35 & $\begin{array}{c}\text { MCA-r, } \\
\text { subcortical }\end{array}$ & 22 & 63 & 0 & rel. \\
\hline P06 & 54 & $\begin{array}{c}\text { MCA-1, } \\
\text { subcortical }\end{array}$ & 60 & 65 & 0 & rel. \\
\hline P07 & 46 & $\begin{array}{c}\text { MCA-r, } \\
\text { cortical }\end{array}$ & 34 & 6 & $1+$ & rel. \\
\hline P08 & 72 & $\begin{array}{c}\text { MCA-r, } \\
\text { cortical }\end{array}$ & 13 & 65 & 0 & rel. \\
\hline & \begin{tabular}{l} 
(flex $)$ \\
\hline
\end{tabular} & & & 0 & \\
\hline
\end{tabular}

were approved by the Medical Ethics Review Committee of the Medisch Spectrum Twente (Enschede, the Netherlands). Measurements with the stroke subjects were performed at the Leiden University Medical Center and were approved by the Medical Ethics Review Committee of the Leiden University Medical Center (Leiden, the Netherlands). All subjects gave signed informed consent before the measurements.

\section{B. Experimental Setup}

Subjects were seated next to a wrist manipulator (Moog Inc., Nieuw-Vennep, the Netherlands), see Fig. 1. The wrist manipulator (WM) is an actuated rotating device with a single degree of freedom that can exert flexion and extension perturbations to the wrist joint. The lower arm of the subject was strapped in an arm rest while the subject held the handle of the WM. The axis of rotation of the WM was aligned with the axis of rotation of the wrist. The lever of the WM is equipped with a force transducer to measure the torques exerted by the subject.

EEG was measured using 64 scalp electrodes, placed according to the 5\% electrode system [22] using a standard EEG cap with $\mathrm{Ag} / \mathrm{AgCl}$ electrodes (measurements at University of Twente (normal subjects): WaveGuard cap by ANT, Enschede, the Netherlands. Measurements at Leiden University Medical Center (stroke subjects): actively shielded headcap by TMSi, Oldenzaal, the Netherlands). Electrode impedances were below $20 \mathrm{kOhm}$ and signal quality was monitored online. The EEG was measured against common average reference. The electromyogram (EMG) was measured from the flexor carpi radialis $\left(\mathrm{EMG}_{\mathrm{FCR}}\right)$ and the extensor carpi radialis $\left(\mathrm{EMG}_{\mathrm{ECR}}\right)$ using bipolar $\mathrm{Ag} / \mathrm{AgCl}$ electrode pairs placed on the muscle belly. In the normal subjects, the vertical electro-oculogram (EOG) was measured to monitor eye blinks, in the stroke subjects the frontal EEG channels (Fp1, Fpz and Fp2) were used to detect eye blinks. All physiological signals were sampled at $2048 \mathrm{~Hz}$ (Refa system by TMSi, Oldenzaal, the Netherlands). 

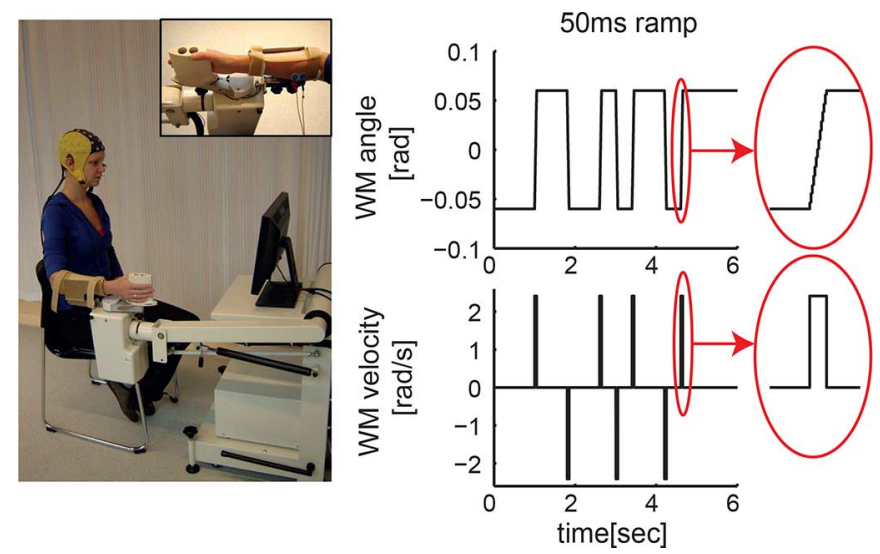

Fig. 1. Left: overview of the experimental setup. Subject holds the lever of the WM and the lower arm is strapped in an arm rest using Velcro straps. To support the subject, visual feedback of the target torque and the exerted torque ( $2 \mathrm{~Hz}$ low-pass filtered with third-order Butterworth filter) are provided on the display in front of the subject. EEG is measured using a head cap (64 channels), bipolar EMG is measured from the flexor carpi radialis (FCR). Handle of the WM moves according to a block shaped reference profile, imposing fast flexion and extension ramp movements on the wrist. Right: portion of the block shaped position perturbation signal with a 50-ms stretch, position profile and velocity profile. During the transition between the two positions the handle has a constant velocity.

The angle of the WM and the torque exerted on the lever were synchronously measured on a separate system at $2048 \mathrm{~Hz}$ (Porti system by TMSi, Oldenzaal, the Netherlands) or via optical isolation modules on the same amplifier as the physiological signal.

\section{Protocol}

1) Normal Subjects: Normal subjects exerted a constant wrist flexion torque on the handle of the WM, while the WM imposed block shaped angular position perturbations. Visual feedback of the exerted and the target torque were presented via a display. Subjects were instructed to keep the exerted torque within a range of $1.8 \pm 0.27 \mathrm{Nm}$. Because the maximum torque the WM could deliver was limited for safety reasons, maximal voluntary contraction torque could not be determined per subject. All subjects reported that this target torque could easily be maintained. For the visualization the exerted torque was filtered online (third-order low-pass Butterworth, $2 \mathrm{~Hz}$ ).

The block-shaped angular position perturbations imposed alternating flexion and extension ramp-and-hold movements on the wrist. During the ramp movement the velocity of the WM was $2.4 \mathrm{rad} / \mathrm{s}$. The interval between ramp movements is chosen pseudo-randomly to ensure an unpredictable perturbation for the subject and was an integer multiple of $400 \mathrm{~ms}$. Combinations of ramp amplitudes and task instructions resulted in three conditions:

1) active 50-ms ramp: stretch duration of $50 \mathrm{~ms}$ during the isotonic force task (amplitude $=0.12 \mathrm{rad}$ );

2) active 25-ms ramp: stretch duration of $25 \mathrm{~ms}$ during the isotonic force task (amplitude $=0.06 \mathrm{rad}$ );

3) relaxed 50-ms ramp: stretch duration of $50 \mathrm{~ms}$ without an active task, subjects held the handle of the WM without applying a force.

These tasks were part of a larger protocol which also included other types of tasks [23]. Subjects were given sufficient rest be- tween parts of the protocol to prevent interference. To restrict the duration of the total protocol, not all subjects performed all tasks: all 22 normal subjects performed the two active conditions; ten normal subjects performed the relaxed condition as an additional task. Furthermore, nine subjects performed the protocol in a second measurement session. The two measurement sessions were separated by at least one week (range: 7-39 days, median: 16 days). For five subjects the second measurement included the relaxed 50-ms ramp task.

In all conditions, five 60 -s trials were recorded with sufficient rest time to prevent fatigue. This results in a total of 360 joint movements. The order of the conditions was fixed and performed as indicated. Subjects performed an active 50-ms ramp trial to practice which was not included in the analysis.

2) Stroke Subjects: Based on the experience from the normal subjects, the protocol for the stroke subjects was slightly adapted. First, the block-shaped angular position perturbation was shortened and adapted. Some of the normal subjects indicated that the perturbation signal with the $50-\mathrm{ms}$ ramps felt aggressive due to the large amplitude of the stretch and the high repetition rate. The ramp velocity was not changed. The position perturbation used with the stroke subjects had a ramp duration of $30 \mathrm{~ms}$ with a constant speed of $2.4 \mathrm{rad} / \mathrm{s}$ (amplitude $=0.072 \mathrm{rad}$ ). The minimal time between ramps was $500 \mathrm{~ms}$. The same angular position perturbation was used in the measurements of all stroke subjects. Second, all stroke subjects performed the task both with the wrist at the affected side and at the unaffected side. At each side, at least 8 trials of $30 \mathrm{~s}$ were performed. This results in a total of 192 joint movements, from the normal subjects clear StrEP waveforms could be obtained with this number of segments. The affected side was measured first to ensure that data was always collected from the affected side even when the complete protocol would be too long for the stroke subject to finish. Third, and finally, after performing the measurements of the first two stroke subjects the task instruction was changed. The active tasks were challenging, also for the stroke subjects with good motor function. Two stroke subjects performed the active isotonic wrist flexion task, the other six stroke subjects were relaxed while they held the handle of the WM that moved according to the position perturbation. Table I indicates which task a stroke subject performed. The same task was performed at the affected and the unaffected side.

\section{Data Analysis}

Recorded signals were processed offline using MATLAB 7.11 (the MathWorks, Inc., Natick, MA, USA). Recorded EEG and EMG was high-pass filtered $(1 \mathrm{~Hz}$, fourth-order Butterworth filter applied with zero phase shift) to remove drift. EMG was rectified and subsequently low-pass filtered $(80 \mathrm{~Hz}$, fourth-order Butterworth filter applied with zero phase shift). All signals were segmented into epochs time locked to the onset of movement of the WM: from $200 \mathrm{~ms}$ before movement onset until $400 \mathrm{~ms}$ post-movement onset.

Segments were visually inspected and segments that contained eye blinks or muscle activity in the EEG were removed. Segments from relaxed task conditions were excluded when EMG activity was seen prior to movement onset. Presence of EMG activity was assessed visually by the variance of the signal prior to movement onset and the presence of action potentials 
StrEP at motor cortex

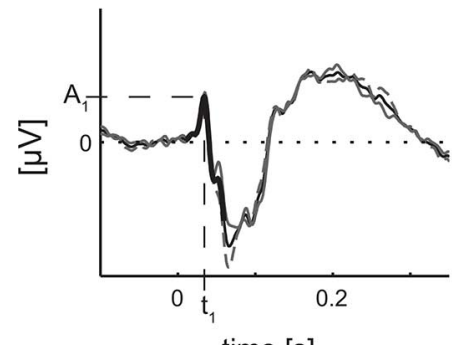

time [s]
StrEP at vertex

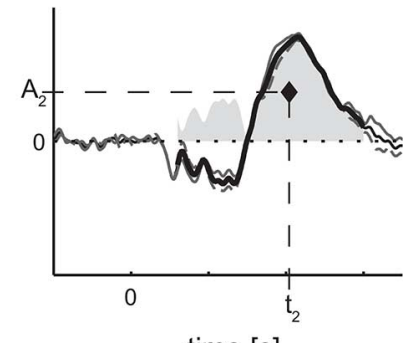

time [s]
Fig. 2. StrEP features. StrEP (solid black line) of a representative normal subject at the contralateral motor cortex (left) and vertex (right) in the active 50-ms ramp condition. Grey lines are the StrEP of the extension (solid gray) and the flexion movements (dashed gray). Early peak latency $\left(t_{1}\right)$ and early peak amplitude $\left(A_{1}\right)$ are determined from the interval 10 to $60 \mathrm{~ms}$ (thicker black line) in the evoked potential at the contralateral motor cortex. Late mean absolute amplitude $\left(A_{2}\right)$ and $50 \%$ late amplitude latency $\left(t_{2}\right)$ are determined from the interval between 60 and $300 \mathrm{~ms}$ (thicker black line and absolute amplitude shaded) in the evoked potential at the vertex.

in the signal. The mean $\mathrm{EMG}_{\mathrm{FCR}}$ stretch response was calculated by averaging the $\mathrm{EMG}_{\mathrm{FCR}}$ over all remaining extension movements.

EEG channels in remaining segments were further filtered using a low-pass filter $(70 \mathrm{~Hz}$, fourth-order Butterworth filter applied in the forward direction) and the $50 \mathrm{~Hz}$ component was removed from each segment using the discrete Fourier transform [24]. Finally, spatial selectivity of the EEG was enhanced by transforming to a nearest neighbor Laplacian derivation, i.e., each EEG channel was referenced to the mean of the four neighboring channels. When the task was performed with the left hand, the EEG channels were mirrored around the midline such that in visualization the hemisphere contralateral to the movement is always the left side.

Before calculating the mean StrEP the baseline of each segment was corrected by subtracting the average potential in the $50 \mathrm{~ms}$ before movement onset from the waveform. At each electrode the mean StrEP was obtained by averaging the baseline corrected waveforms over all segments. Note that the averaging includes both the flexion and the extension movements. By averaging over both flexion and extension movements the number of available segments for a single StrEP waveform doubles which reduces the influence of random fluctuations on the StrEP waveform. In preliminary analysis of the StrEP waveforms a high cross correlation coefficient was found between StrEPs obtained from flexion and extension segments. In the active 50-ms task cross correlation was 0.73 at the contralateral motor cortex and 0.87 at the vertex. In the active $25-\mathrm{ms}$ task cross correlation was 0.74 at the contralateral motor cortex and 0.89 at the vertex. In the relax $50-\mathrm{ms}$ task the cross correlation was 0.86 at the contralateral motor cortex and 0.87 at the vertex. These high cross correlations indicate that flexion and extension StrEP waveforms are highly similar (see also Fig. 2) and can be averaged to obtain a less noisy estimate of the StrEP waveform.

Two electrode positions were chosen for further evaluation of the StrEP waveform. The waveform at electrode C3 (C4 when the task was performed with the left wrist) was evaluated because in normal subjects, the early peak (within $60 \mathrm{~ms}$ after movement onset) was largest at this location. This location is referred to as contralateral motor cortex. In addition, the waveform at the vertex $(\mathrm{Cz})$ was evaluated because in normal subjects, the overall largest peak was measured at this location.
1) Comparison of StrEP Waveforms: In the normal subjects, similarity of StrEP waveforms was evaluated by calculating the cross correlation coefficient $(0<t<400 \mathrm{~ms})$ between conditions and sessions for both the contralateral motor cortex and the vertex.

In the stroke subjects, similarity between the affected and unaffected side was evaluated by the cross correlation coefficient between the StrEP waveforms elicited by affected side movement and unaffected side movement. A Wilcoxon rank-sum test was used to compare cross correlation coefficients between the stroke subjects with good motor function and with poor motor function $(\alpha=0.05)$.

2) Evaluation of StrEP Features: Specific features of the StrEP (Fig. 2) were further assessed as follows. In the waveform at the contralateral motor cortex the presence, amplitude and latency of the early peak was determined. The early peak was considered present if the maximum positive deflection at the contralateral motor cortex in the interval between 10 and $60 \mathrm{~ms}$ after movement onset exceeding the $99 \%$ confidence interval (CI): $E P_{C 3}(t)-C I_{C 3}(t)>0$ using

$$
C I_{C 3}(t)=2.6 \frac{1}{\sqrt{n}} \sigma_{E P_{C 3}}(t) .
$$

The standard deviation over repetitions of the averaged StrEP $\left(\sigma_{E P}(t)\right)$ was divided by the square root of the number of segments $(n)$ to obtain the standard error of the mean. This was multiplied by 2.6 to obtain the $99 \%$ CI. Only if the early peak was larger than the $99 \%$ CI, i.e., the peak was present, the early peak latency $\left(t_{1}\right)$ and early peak amplitude $\left(A_{1}\right)$ were determined.

As later peaks stretch over a longer time interval, we used the mean absolute amplitude in the late interval at the vertex instead of considering individual peaks. The amplitude of the late peaks (late mean amplitude, $A_{2}$ ) was determined as the mean absolute amplitude in the late interval $(60<t<300 \mathrm{~ms})$. The latency of the late peaks ( $50 \%$ late amplitude latency, $\left.t_{2}\right)$ was determined as the latency at which the cumulative absolute amplitude was $50 \%$ of the total cumulative absolute amplitude in the late interval.

In the normal subjects, the effect of condition, i.e., on StrEP features, was assessed by linear mixed model analysis, as this technique is particularly suited for unbalanced datasets [25]. For each StrEP feature, a model was fitted with the condition as a fixed effect factor and a variable intercept to allow inter-individual differences between the subjects. When a significant effect of the condition on the StrEP feature was found, estimated marginal means were used to test for differences between the active $50-\mathrm{ms}$ ramp and the relaxed $50-\mathrm{ms}$ ramp and between the active 50-ms ramp and the active $25-\mathrm{ms}$ ramp. Bonferroni correction for multiple comparisons was applied. Linear mixed model analyses were performed with IBM SPSS Statistics version 20. Reliability of the StrEP features over the two sessions was evaluated using the intraclass correlation coefficient $(\operatorname{ICC}(2,1))$.

Absolute difference between the StrEP features of affected and unaffected hemisphere of stroke subjects with good motor function were compared to subjects with poor function using a Wilcoxon rank-sum test. 

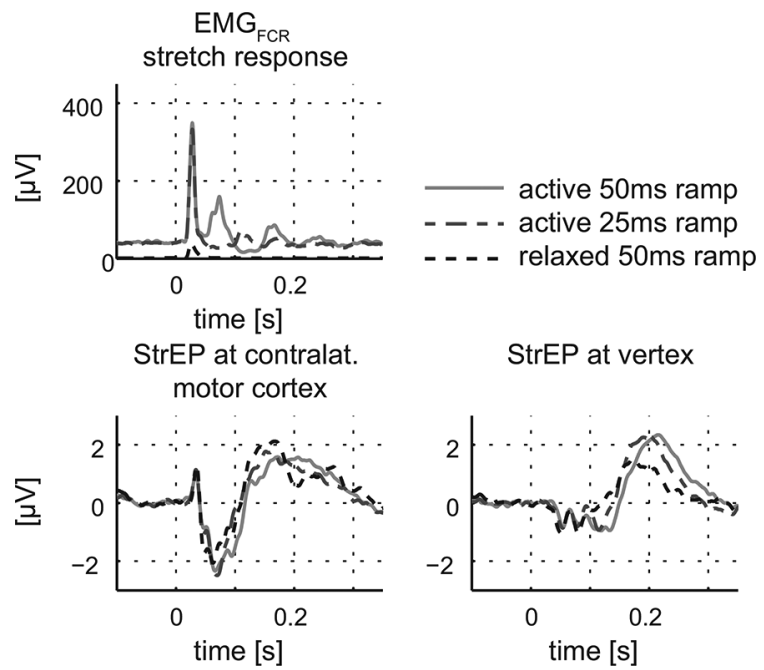

Fig. 3. Stretch responses in the FCR muscle and StrEPs in a representative normal subject that performed the three task conditions. All traces are from the same measurement session. Cross correlation coefficients between active 50 -ms ramp and active $25-\mathrm{ms}$ ramp conditions: 0.93 at the contralateral motor cortex and 0.90 at the vertex. Cross correlation coefficients between the active and relaxed 50-ms ramp conditions: 0.89 at the contralateral motor cortex and 0.74 at the vertex.

\section{RESULTS}

\section{A. Normal Subjects}

The extension movements elicited typical stretch responses in the FCR muscle in the normal subjects (see Fig. 3). Typically, in the active $25-\mathrm{ms}$ ramp condition, a single short latency response (within $50 \mathrm{~ms}$ after movement onset, mean latency : $30 \mathrm{~ms}$, std: $3 \mathrm{~ms}$ ) was elicited. In the active $50 \mathrm{~ms}$ the short latency response (mean latency: $30 \mathrm{~ms}$, std: $4 \mathrm{~ms}$ ) had an amplitude similar to the active 25-ms ramp condition. However, the longer ramp also elicited a longer latency response (between 50 and $150 \mathrm{~ms}$ after movement onset, mean latency: $67 \mathrm{~ms}$, std: $7 \mathrm{~ms}$ ). The relaxed 50-ms ramp condition elicited a single short latency response (mean latency: $32 \mathrm{~ms}$, std: $3 \mathrm{~ms}$ ) with an amplitude that was considerably smaller than in the active conditions.

In normal subjects, fast wrist movement elicited consistent StrEPs in all conditions. The StrEPs were mainly localized over the contralateral sensorimotor area with the early peak (within the $60 \mathrm{~ms}$ after movement onset) having the largest amplitude at electrode C3 (Fig. 4).

The active 50-ms ramp and active 25-ms ramp elicited StrEPs with a similar waveform (see Fig. 3 ). The average cross correlation coefficient between the StrEPs in the active 50-ms condition and the active $25-\mathrm{ms}$ ramp condition was 0.85 at the contralateral motor cortex and 0.92 at the vertex. Also in the active 50-ms ramp condition and the relaxed 50-ms ramp condition there were large similarities between StrEP waveforms, with an average cross correlation coefficient of 0.83 at the contralateral motor cortex and 0.76 at the vertex.

There was also a large similarity of the StrEP waveforms over sessions. The average cross correlation coefficient between the same conditions in the first and the second measurement session was 0.91 at the contralateral motor cortex and 0.93 at the vertex.
1) Variability of StrEP Features: The active $25-\mathrm{ms}$ condition elicited a significant early peak in all subjects and both sessions. The active 50-ms ramp condition also elicited a significant early peak in most subjects ( 21 out of 22 subjects in the first session and eight out of nine subjects in the second session). The relaxed 50-ms ramp condition elicited a significant early peak in all normal subjects that performed this condition in the first measurement (ten subjects). However, in the second measurement session the relaxed 50-ms ramp condition elicited a significant early peak in only two out of the five subjects that performed in task in the second session.

The averages of the StrEP features in the different conditions and sessions are listed in Table II. There was a significant effect of condition on the early peak amplitude $(F(2,48.364)=$ $8.341, p=0.001)$. The early peak amplitude was on average $0.29 \mu \mathrm{V}$ larger in the relaxed 50-ms ramp condition than in the active 50-ms ramp condition according to post hoc comparisons $(p<0.001)$. There was no effect of condition on the early peak latency $(F(2,49.193)=0.69, p=0.506)$ and the effect of condition on the late mean amplitude was on the border of significance $(F(2,54.345)=3.091, p=0.054$. Finally, there was a significant effect of condition on the $50 \%$ late amplitude latency $(F(2,57.675)=4.524, p=0.015)$. The average $50 \%$ late amplitude latency was $11 \mathrm{~ms}$ shorter in the relaxed $50-\mathrm{ms}$ ramp condition compared to the active 50 -ms ramp condition $(p=0.017)$.

Reliability of both StrEP features relating to amplitude was very good: early peak amplitude ICC $=0.94$, late mean amplitude $\mathrm{ICC}=0.85$. The reliability of the StrEP features relating to time was good for the early peak latency $\mathrm{ICC}=0.78$. However, the reliability was poor for the $50 \%$ late amplitude latency: $\mathrm{ICC}=0.29$.

\section{B. Stroke Subjects}

The $\mathrm{EMG}_{\mathrm{FCR}}$ at the affected side of P11 was excluded because the signal contained large artefacts. The extension movements elicited stretch responses in the FCR muscles of the stroke subjects that performed the active task (P01 and P02, Fig. 5, latency $42 \mathrm{~ms}$ for P01, latency $31 \mathrm{~ms}$ for P02). Stretch responses of the affected side FCR muscle were also elicited in the stroke subjects with poor motor function (P03 latency $35 \mathrm{~ms}$, P04 latency $41 \mathrm{~ms}$ and P07 latency $42 \mathrm{~ms}$ ), although these subjects were relaxed. These subjects also experienced some spasticity of the wrist as determined by the Ashworth scale [26] (Table I). The muscle stretch responses were clearly visible in the EMG and consisted of a short latency muscle stretch response (Fig. 5).

The quality of the EEG data recorded from the stroke subjects was poorer than in the normal subjects and more segments were rejected due to eye blinks or EMG artefacts. In the normal subjects on average $10 \%$ of the segments was rejected due to artefacts, in the stroke subjects on average $35 \%$ of the segments was rejected. Stroke subject $\mathrm{P} 04$ showed continuous muscle activity on channels $\mathrm{C} 3$ and $\mathrm{C} 4$ and on surrounding temporal electrodes, resulting in a very low signal-to-noise ratio, these channels were therefore excluded from analysis. 

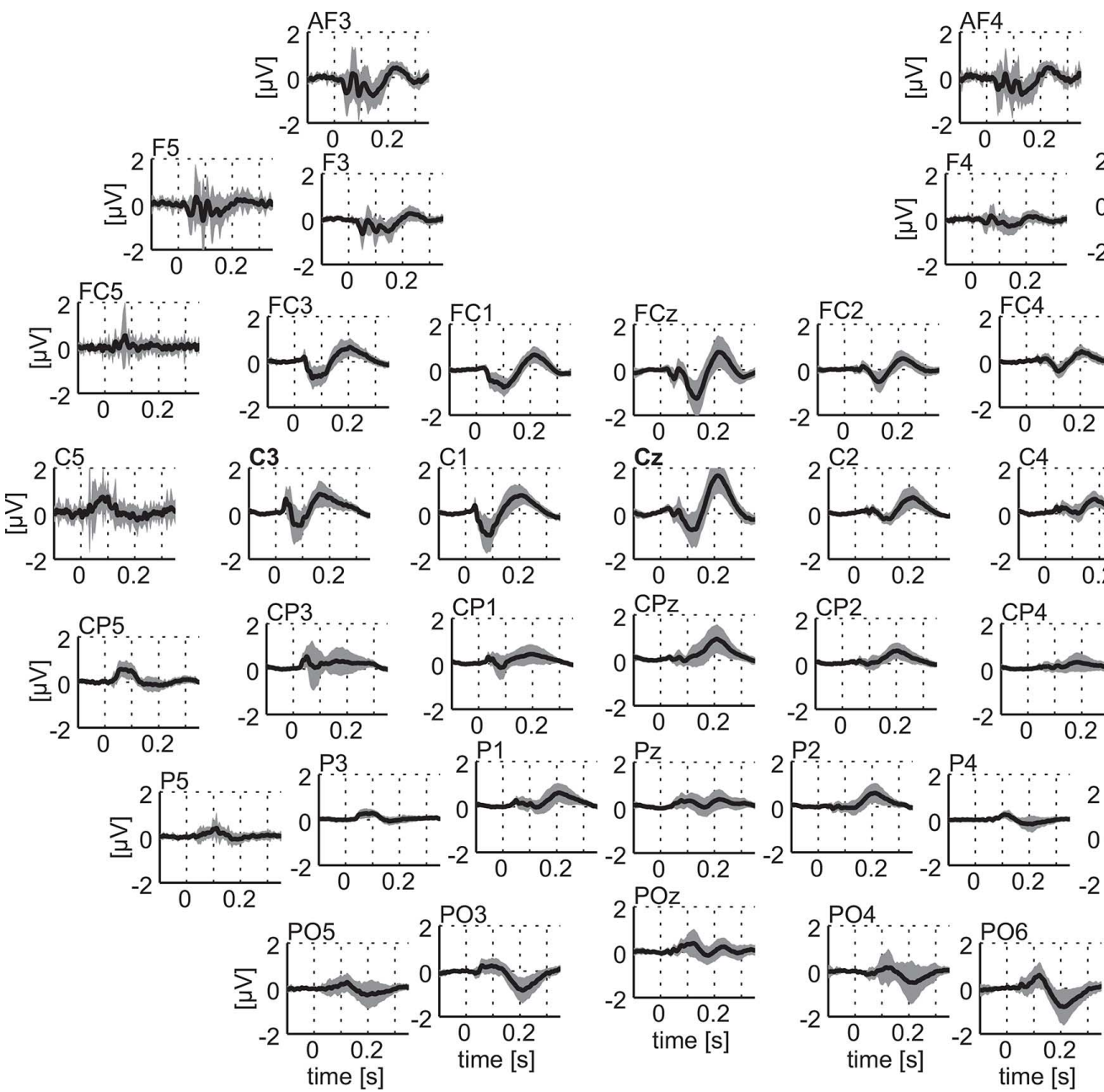

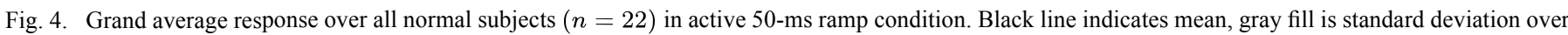

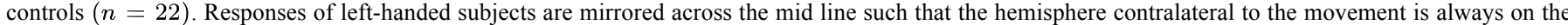
left side.

TABLE II

Averages and Standard Deviations of StrEP Features in Different TASKS AND Sessions In Normal SubJects. S1: SEsSions 1, S2: SEsSion 2. $A_{1}$ : Early PeaK Amplitude, $A_{2}$ : Late Mean Amplitude, $t_{1}$ : Early Peak Latency, $t_{2}: 50 \%$ Late Amplitude Latency. Not All Normal SubJects Showed Significant Early PeaK. $n$ Is Number of SubJects on Which Estimate of AVerage and Standard DeViation Is Based, Between Square Brackets the Number of Normal SubJects THAT PERFORMED TASK

\begin{tabular}{ccccc}
\hline & \multicolumn{2}{c}{$A_{1}[\mu V]$} & \multicolumn{2}{c}{$A_{2}[\mu V]$} \\
& $\mathrm{S} 1$ & $\mathrm{~S} 2$ & $\mathrm{~S} 1$ & $\mathrm{~S} 2$ \\
\cline { 2 - 5 } active 50ms ramp & $0.86 \pm 0.59$ & $0.85 \pm 0.58$ & $0.85 \pm 0.38$ & $0.91 \pm 0.47$ \\
& $n=21[22]$ & $n=8[9]$ & $n=22[22]$ & $n=9[9]$ \\
active 25ms ramp & $0.87 \pm 0.64$ & $0.89 \pm 0.73$ & $0.79 \pm 0.40$ & $0.80 \pm 0.47$ \\
& $n=22[22]$ & $n=9[9]$ & $n=22[22]$ & $n=9[9]$ \\
relaxed 50ms ramp & $0.93 \pm 0.72$ & $2.03 \pm 0.92$ & $0.70 \pm 0.52$ & $0.83 \pm 0.45$ \\
& $n=10[10]$ & $n=2[5]$ & $n=10[10]$ & $n=5[5]$ \\
& \multicolumn{5}{c}{$t_{1}[m s]$} & \multicolumn{2}{c}{$t_{2}[m s]$} \\
active 50ms ramp & $40 \pm 6.6$ & $38 \pm 7.6$ & $199 \pm 13$ & $200 \pm 11$ \\
& $n=21[22]$ & $n=8[9]$ & $n=22[22]$ & $n=9[9]$ \\
active 25ms ramp & $40 \pm 6.3$ & $37 \pm 7.4$ & $194 \pm 14$ & $188 \pm 19$ \\
& $n=22[22]$ & $n=9[9]$ & $n=22[22]$ & $n=9[9]$ \\
relaxed 50ms ramp & $39 \pm 3.8$ & $46 \pm 6.6$ & $184 \pm 14$ & $196 \pm 13$ \\
& $n=10[10]$ & $n=2[5]$ & $n=10[10]$ & $n=5[5]$ \\
\hline
\end{tabular}
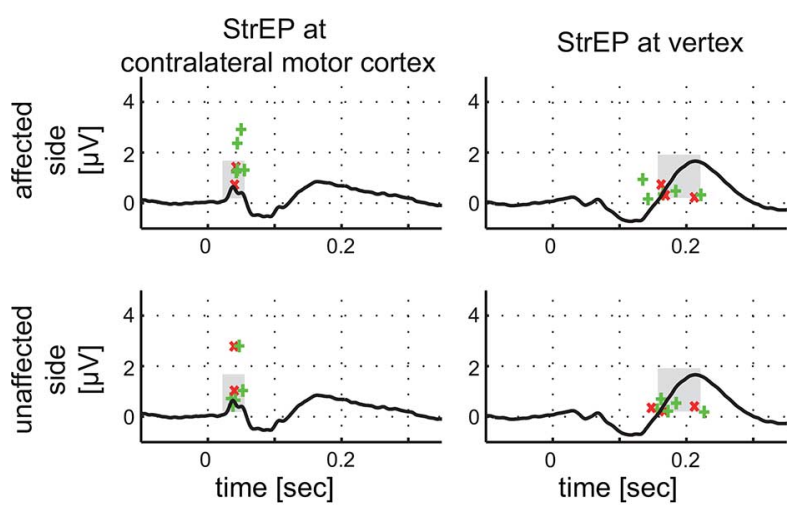

+ StrEP feature good motor function

$\times$ StrEP feature poor motor function

- grand average normal subjects range StrEP feature

in normal subjects

Fig. 5. Individual FCR stretch responses and StrEPs of the stroke subjects. A green block indicates a subject with good motor function, a red block indicates poor motor function. Left column denotes muscle stretch response, middle column the response at the contralateral motor cortex (i.e., C3 or C4), right column the response at the vertex $(\mathrm{Cz})$. As a reference the grand average StrEP and standard deviation (std) of the normal subjects in the active 50-ms ramp condition is plotted. No contralateral motor cortex StrEPs are plotted for P04, this data was contaminated by continuous scalp muscle activity. Affected side $\mathrm{EMG}_{\mathrm{FCR}}$ of P11 was excluded due to artefacts. 


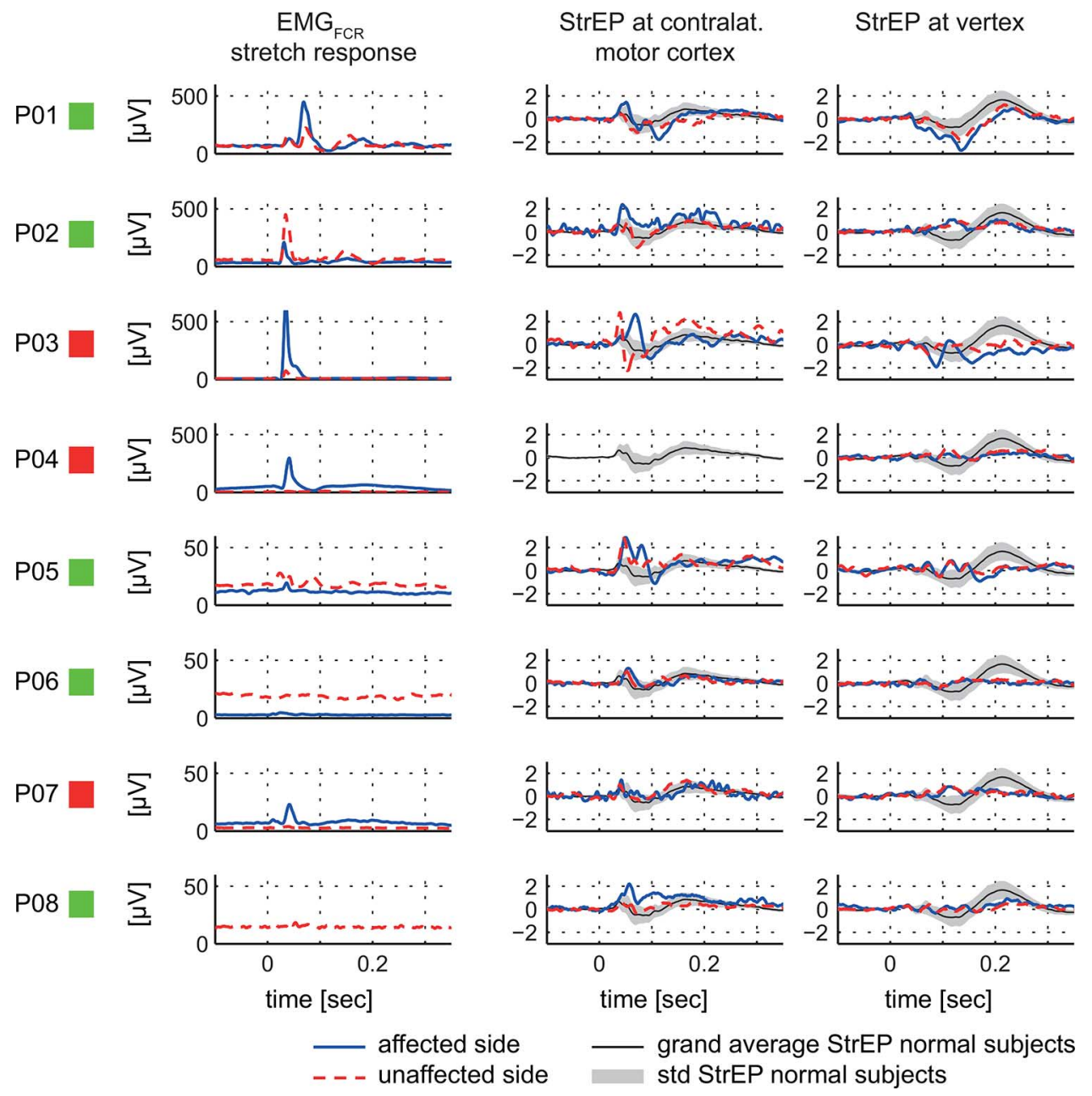

Fig. 6. StrEP features in stroke subjects. Left: StrEP at the contralateral motor cortex with the early peak $\left(t_{1}, A_{1}\right)$ indicated for individual stroke subjects and the range of $t_{1}$ and $A_{1}$ found in normal subjects over all conditions and sessions. Right: StrEP at the vertex with the late amplitude features $\left(t_{2}, A_{2}\right)$ indicated for individual stroke subjects and the range of $t_{2}$ and $A_{2}$ found in normal subjects over all conditions and sessions.

Fig. 5 shows the individual StrEPs of the stroke subjects in response to affected and unaffected wrist movement. This figure shows that there is considerable inter-individual variation in StrEP waveforms and that the stroke subjects have different StrEP waveforms compared to the normal subjects, especially at the vertex. All stroke subjects presented a significant early peak at the contralateral motor cortex within the first $60 \mathrm{~ms}$ after movement onset, both in the StrEP elicited by movement at the affected and by movement at the nonaffected side. Especially in the later interval (between 60 and $300 \mathrm{~ms}$ after movement onset) and at the vertex, the shape of the StrEP waveform in stroke subjects differs from the typical StrEP waveform of the normal subjects. In this interval, the normal subjects grand average StrEP at the vertex is characterized by a negative deflection followed by a larger positive deflection peaking around $200 \mathrm{~ms}$ after movement onset. This large positive deflection was absent in most stroke subjects. Several stroke subjects (P02, P04, P05 and P07) showed a positive deflection around $100 \mathrm{~ms}$. Only P01 showed StrEPs which resembled the global shape of the StrEPs seen in normal subjects at both affected and nonaffected side. Although this stroke subject had a good motor function, other subjects with good motor function did not necessarily present a close to normal StrEP waveform.
The early peak latency $\left(t_{1}\right)$ of the StrEPs of the stroke subjects fell in the same range as seen in the normal subjects (Fig. 6). However, the early peak amplitude $\left(A_{1}\right)$ exceeded the range found in normal subjects in three stroke subjects. P05 showed a high early peak amplitude on both sides, $\mathrm{P} 02$ and $\mathrm{P} 03$ had a high early peak amplitude at the affected and unaffected side respectively.

For all stroke subjects, the late mean amplitude $\left(A_{2}\right)$ was low compared to the range of values found in the normal subjects (Fig. 6). In P06 the late mean amplitude on the affected side was below the range seen in normal subjects. In P08 the late mean amplitude on the unaffected side was below the range of normal subjects.

In the stroke subjects with good motor function there was a considerable similarity between the StrEP waveforms elicited at the affected and the unaffected side. In the stroke subjects with poor motor function, the similarity between affected and unaffected hemisphere tended to be lower (Fig. 7, left side). However, no statistical significant difference was found between the good and poor function stroke subjects (Wilcoxon rank-sum test: $p=0.19$ at the contralateral motor cortex, $p=0.14$ at the vertex). 

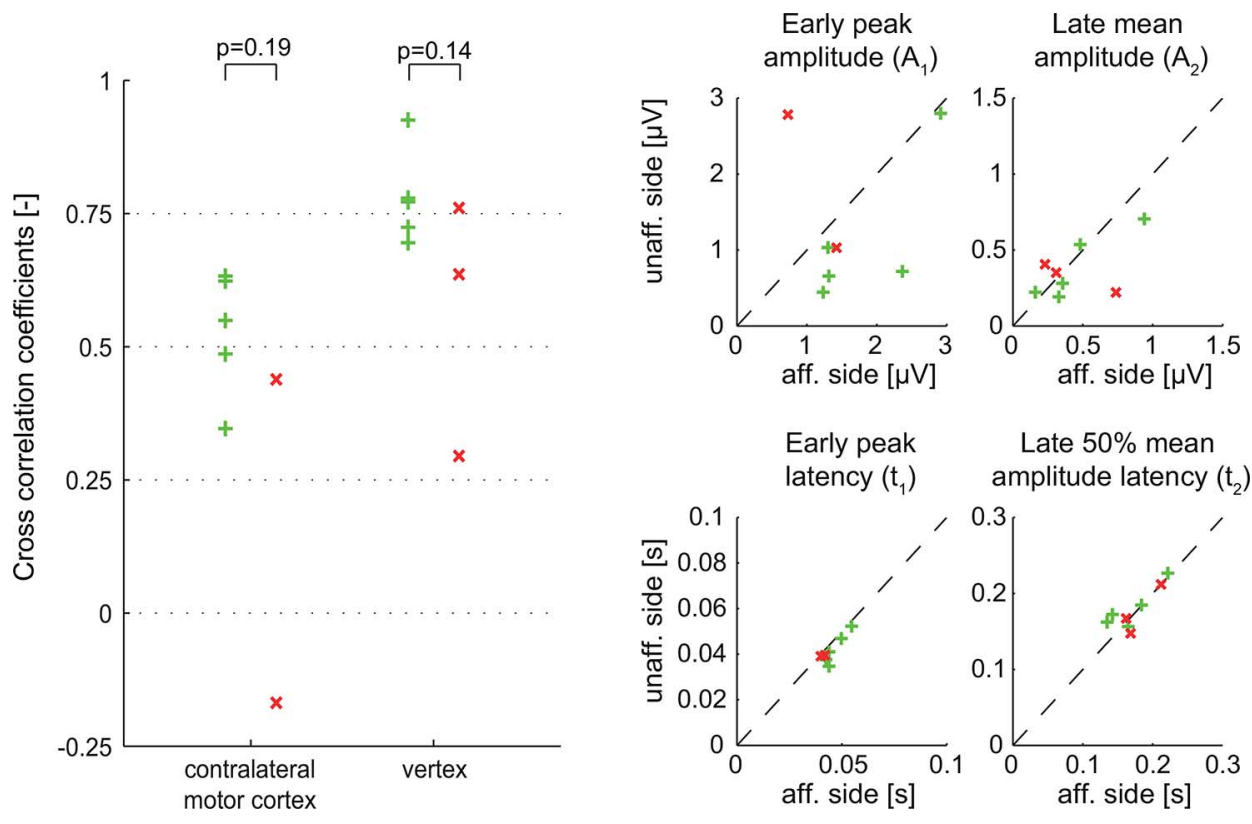

Fig. 7. Left: cross correlation coefficients between StrEP waveforms elicited at the affected and the unaffected side in stroke subjects. Right: StrEP features measured at the affected (x-axes) and the unaffected side (y-axes). Dashed line indicates the line $y=x$. A green " + " indicates a subject with good motor function, a red " $x$ " indicates a subject with poor motor function.

The StrEP latency features (Fig. 7, right side) were similar at the affected and unaffected side (early peak latency ICC $=$ $0.73,50 \%$ late amplitude latency ICC $=0.84$ ). The amplitude features showed a larger degree of variation between the affected and unaffected side (early peak amplitude ICC $=0.19$, late mean amplitude: ICC $=0.53$ ). There was no significant difference between stroke subjects with good and poor motor function in the absolute difference between StrEP features at the affected and the unaffected side (Wilcoxon rank-sum test: $p=0.57$ for the early peak amplitude, $p=0.19$ for the early peak latency, $p=0.79$ for the late mean amplitude and $p=0.46$ for $50 \%$ late amplitude latency).

\section{DISCUSSION}

In this study we evaluated the cortical response to fast muscle stretch: StrEP. The StrEP waveform of normal subjects is characterized by an early peak within $60 \mathrm{~ms}$ after movement onset over the contralateral primary motor cortex, and a complex of late peaks between 60 and $300 \mathrm{~ms}$ after movement onset at the vertex. In stroke survivors the early peak was also present. However, they showed abnormal late peaks both in the StrEP elicited by affected and by unaffected wrist movement.

\section{A. Consistent Stretch Evoked Potentials in Normal Subjects}

The 25- and 50-ms ramps elicited clear muscle stretch responses in the active conditions. The $25-\mathrm{ms}$ ramp only elicited a short latency response (within $50 \mathrm{~ms}$ after movement onset) while the 50-ms ramp elicited both a short and a long latency stretch response (between 50 and $150 \mathrm{~ms}$ after movement onset), this result is consistent with previous experimental work using a similar experimental setup [27]. The short latency response is generally thought to be mediated by a monosynaptic reflex pathway. The origin of the long latency reflex is more complex, several pathways may contribute to this reflex including a transcortical pathway [16], [28], [29].

Although the muscle stretch response recorded by the EMG depends on the direction of joint movement, StrEP waveforms obtained from flexion and extension movements were very similar (Fig. 2). This suggests that the responses to flexion and extension movements are generated by cortical populations of neurons that are so close that the EEG cannot distinguish between them. A potential effect of direction on StrEP features has been lost by collapsing over movement direction, such an effect could be caused by a difference in sensitivity of muscles spindles between the active wrist flexion muscles and the passive wrist extension muscles in the active tasks. However, averaging over both flexion and extension movements doubles the number of available segments and substantially increases the signal-to-noise ratio of the StrEP [17]. As the signal-to-noise ratio of the EEG data from the stroke subjects could be very poor, averaging over flexion and extension movements allows including more segments without increasing the total recording time for the subjects.

Within a single subject the StrEP waveform was very similar over the two stretch amplitudes (mean cross correlation $>0.85$ ) and also when comparing StrEPs during the active conditions (i.e., an isotonic wrist flexion task) and the relaxed conditions (mean cross correlation $>0.76$ ). In all task conditions the StrEP waveform is characterized by an early peak at a latency of 40 ms after movement onset, localized at the sensorimotor cortex contralateral to the wrist movement. In the later interval (between 60 and $300 \mathrm{~ms}$ after movement onset) the StrEP has the largest amplitude at the vertex and is characterized by an initial negative deflection, followed by a positive deflection, peaking at around $200 \mathrm{~ms}$ after movement onset (Fig. 3). The global StrEP waveform is in concordance with the waveforms elicited 
by muscle stretch in previous studies, although detailed comparison of latency and amplitude features is hampered by differences in the type of perturbations applied, EEG derivation and data processing [15]-[17], [28].

The active $25-\mathrm{ms}$ ramp condition elicited a significant early peak in all normal subjects, while the 50-ms conditions did not elicit this peak in some subjects. This suggests that shorter ramp movements may be more suitable for eliciting the early peak of the StrEP. It was previously reported that an early peak, between 30 and $50 \mathrm{~ms}$, after movement onset was not consistently present after passive movement of at least $100 \mathrm{~ms}$ of the index finger [17].

Despite the differences in elicited muscle stretch reflexes, the two ramp amplitudes did not result in significant difference between StrEP features. Processes leading to the muscle stretch response and the StrEP occur partly via the same pathway carrying sensory input from muscle spindles and Golgi tendon organs to the spinal cord. However, the StrEP involves the entire afferent sensory pathway including the cortex, while the muscle stretch response involves the spinal reflex pathways. Neural networks resulting in the modulation of the muscle stretch response are not necessarily part of the afferent sensory pathway eliciting the StrEP. The different pathways involved in muscle stretch response and StrEP could explain the difference in sensitivity to perturbation characteristics like the ramp amplitude. A model simulation study previously showed that also the monosynaptic Ia afferent pathway can explain the appearance of long latency responses in the muscle stretch reflexes as a function of stretch duration [27].

StrEP features were sensitive to small changes in the StrEP waveform in the different task conditions: the initial peak amplitude was larger and the $50 \%$ late amplitude latency shorter in the relaxed compared to the active 50-ms ramp condition. Previous studies obtained varying results on changes of StrEP features due to different task instructions. Abbruzzese et al. [15] showed covariation of long latency muscle responses and early StrEP amplitudes (30-75 ms after wrist extension onset) with task instruction. Intention related modulation of early StrEP amplitudes ( $45 \mathrm{~ms}$ after wrist extension onset) was also shown by Spieser et al. [28]. In contrast to these studies, MacKinnon et al. [16] found that StrEPs elicited during tasks with different instruction were only different more than $135 \mathrm{~ms}$ after wrist extension onset, which does not support a relation between a transcortical reflex loop and the StrEP.

While the results on modulation of StrEP features with task instruction are inconclusive, the electrically elicited SSEP is sensitive to differences in tasks. Some of the earliest components of the SSEP are suppressed during active and passive movement, as compared to a resting condition [30], [31]. This effect is known as "gating" and represents the modulation of the sensitivity of the cortex to various, competing sources of sensory information. However, in the SSEP paradigms the different task instructions introduce input to sensory modalities that are inactive in the resting condition. In the StrEP paradigm, the type of sensory input, i.e., movement and torque, is the same in the different tasks; no other sources of sensory input are added. The modulation of StrEP features could therefore represent a different mechanism.
An important aspect of the usefulness of the StrEP as a potential clinical measure for proprioceptive function after stroke is the reliability of the StrEP waveform and features over multiple sessions [32]. StrEP waveforms were very similar over two sessions with between session cross correlations being larger than the between tasks cross correlations. Generally, the reliability of the StrEP amplitude features (early peak amplitude and late mean amplitude) was very good. However, the reliability of the early peak is limited due to its inconsistent presence in the 50-ms ramp tasks. Subjects could even present the peak in one session and not in the second session. The active 25 -ms task did always elicit the early peak. Most suitable to differentiate between subjects thus seems to be the late mean amplitude, this feature can always be extracted from the StrEP and the between session variance is small compared to the variance between sessions.

\section{B. Stretch Evoked Potentials After Hemispheric Stroke}

The possibility to elicit StrEPs during a relaxed task similar to those during an active task opens the possibility to investigate processing of proprioceptive input also in stroke survivors that are not able to voluntarily generate force. Especially in this severely impaired patient group, prediction of the potential motor recovery is difficult [33]. Given the importance of sensory feedback for voluntary motor control and motor learning, assessment of proprioceptive function and sensorimotor integration may aid in the prediction of the potential motor recovery. Such assessment is needed early after stroke, within four weeks post stroke, as recovery in this time window predicts the recovery of a patient after six months [34]. Monitoring recovery of sensorimotor function in these first weeks after stroke will potentially be most valuable and our results show that the StrEP can be elicited within this time frame in subjects with poor motor function.

Fast wrist movement elicited StrEPs both in stroke subjects with good motor function $(\mathrm{FM}>60)$ and with poor motor function $(\mathrm{FM}<30)$. Despite the large heterogeneity in subject characteristics and StrEP waveforms, all stroke subjects showed the early peak at the contralateral motor cortex within $60 \mathrm{~ms}$ after movement onset. This peak most likely represents the arrival of afferent sensory information from the muscle spindles to the motor cortex [16]-[18]. The presence of the early peak shows intact afferent pathways in all stroke subjects, which is in line with the clinical assessment of sensory function Nottingham sensory assessment scale.

While it is likely that the early peak of the StrEP indicates the arrival of afferent sensory information, the meaning of the late peaks is unknown but possibly indicates the processing of proprioceptive input from the muscle spindles by a cortical sensorimotor network. The large variety of StrEP waveforms in the stroke subjects then indicates changes of this sensorimotor network after stroke. While abnormal StrEP waveforms were also seen in stroke subjects with good motor function, changes in the sensorimotor network do not necessarily represent poor motor control. In subacute stroke survivors, StrEP waveform is altered but does not allow discrimination between good and poor motor function.

An unexpected result is that although stroke affects one hemisphere, the StrEP elicited by unaffected wrist movement was 
not necessarily similar to the StrEPs of the normal subjects. Although the StrEP waveforms at the vertex and the contralateral motor cortex were abnormal both in subjects with good and poor motor function, stroke subjects with good motor function tended to have a larger similarity between StrEPs elicited at the affected and unaffected side. However, no significant difference was found in our small group of stroke subjects. A recent study [35] showed that in chronic stroke subjects, recovery is not associated with the absolute normality of beta band EEG power and corticomuscular coherence during muscle contraction but rather with the symmetry of these measures between the ipslesional and contralesional hemisphere. It may be more valuable to compare stroke subjects with themselves instead of healthy controls, as recovery may not result in a restoration of the pre-stroke activity pattern but rather with a new balance between both hemispheres. In our results, the cross correlation between StrEP waveforms seemed to be smaller in the stroke subjects with poor recovery but the difference did not reach statistical significance. Although we cannot confirm this from the current data set, it is expected that the StrEP, like the SSEP, in normal subjects exhibit a large degree of similarity [36].

1) Study Limitations and Future Directions: Quantitative comparison of StrEP features between normal and stroke subjects in this study is hampered due to nonmatched characteristics of the two populations. Subject age can affect the expected latencies of evoked potential peaks due to decreased fibre conduction velocity [37]. Within our study, such conduction velocity related effects would mainly result in increased initial peak amplitude latencies; an overall alteration of evoked potential waveform, like we saw in the stroke subjects, is not expected. One study evaluated age-related changes of evoked potentials induced by joint movement [14]. In that study, cortical potentials induced by ankle flexion did not change as a function of age. However, with only ten subjects, their study population was relatively small. Despite the age difference between the normal and stroke subjects, we found that the stroke subjects all present the initial peak at latencies comparable to the normal, younger, subjects. We did see a large variety in StrEP waveforms in the late interval in the stroke subjects. Although we cannot rule out that age differences contributed to the observed abnormal StrEP waveforms in stroke subjects, age differences are not likely to be the sole explanation.

The difference in task instruction and perturbation characteristics also hampers the direct comparison between normal and stroke subjects. However, like the age differences, these changes in the protocol are not expected to fully explain the abnormal StrEP waveform seen in the stroke subjects. In the normal subjects, the shortening of the ramp duration increased the likelihood of eliciting a significant early peak. Although the initial peak amplitude and the 50\% late amplitude latency differed between the active and relaxed task, the correlation coefficients were high between StrEPs obtained in active and relaxed conditions (average cross correlations $>0.75$ ) indicating that the global StrEP waveform did not change between conditions.

Further assessment of the relation between the StrEP and sensorimotor function after stroke requires data of a larger cohort of patients and matched controls, including patients with good and poor motor function and with good and poor sensory function. Future work should include the longitudinal assessment of StrEP and sensorimotor function in a large cohort of patients from the subacute until the chronic phase to monitor changes in cortical processing of sensory input in relation to recovery. As clinical assessment of sensory function is shown to be unreliable [38], proprioceptive function may be evaluated with objective and quantitative assessment of position sense by robotic devices [39]. Eliciting StrEPs can easily be combined with such robotic assessment of position sense or with robotic rehabilitation training. Comparison of the StrEP with the electrically elicited SSEP in such a longitudinal study design should provide insight into how the cortical response to different types of sensory input is related to sensorimotor integration and recovery of motor function.

\section{Conclusion}

Measurement of cortical responses evoked by fast joint movement (StrEPs) potentially offers a method to objectively measure the integrity of the afferent sensory pathways associated with proprioceptive function and sensorimotor function. In normal subjects, StrEPs have a very consistent waveform across conditions and sessions. With this property the StrEP meets an important prerequisite for clinical value. The peaks at different latencies allow separation between the arrival of input at the cortex and subsequent processing of this information. Measurement of the StrEP is feasible in subacute stroke patients and our first exploration of StrEPs in this patient group indicates that even subjects with good motor function may show abnormal StrEP waveforms. We found no clear association between StrEP features and good or poor motor function in this population, leaving the relation between StrEP features and clinical phenotype to be established.

\section{ACKNOWLEDGMENT}

The authors thank S. Zandvliet for her assistance during the measurements at the Leiden University Medical Center.

\section{REFERENCES}

[1] G. Kwakkel, B. Kollen, and E. Lindeman, "Understanding the pattern of functional recovery after stroke: Facts and theories," Restorative Neurol. Neurosci., vol. 22, no. 3-5, pp. 281-299, Jan. 2004.

[2] L. A. Connell, N. B. Lincoln, and K. A. Radford, "Somatosensory impairment after stroke: Frequency of different deficits and their recovery," Clin. Rehabil., vol. 22, no. 8, pp. 758-767, Aug. 2008.

[3] S. H. Scott, "Optimal feedback control and the neural basis of volitional motor control," Nature Rev. Neurosci., vol. 5, no. 7, pp. 532-46, Jul. 2004.

[4] S. M. Schabrun and S. Hillier, "Evidence for the retraining of sensation after stroke: A systematic review," Clin. Rehabil., vol. 23, no. 1, pp. 27-39, Jan. 2009.

[5] M. J. A. M. van Putten, "The N20 in post-anoxic coma: Are you listening?," Clin. Neurophysiol.: Official J. Int. Fed. Clinical Neurophysiology, vol. 123, no. 7, pp. 1460-1464, Jul. 2012.

[6] J. E. Desmedt and C. Tomberg, "Mapping early somatosensory evoked potentials in selective attention: Critical evaluation of control conditions used for titrating by difference the cognitive P30, P40, P100 and N140," Electroencephalography Clin. Neurophysiol., vol. 74, no. 5, pp. 321-346, 1989.

[7] R. C. Josiassen, C. Shagass, R. A. Roemer, S. Slepner, and B. Czartorysky, "Early cognitive components of somatosensory event-related potentials," Int. J. Psychophysiology: Official J. Int. Organization Psychophysiol., vol. 9, no. 2, pp. 139-149, Sep. 1990. 
[8] T. Tsumoto, N. Hirose, S. Nonale, and M. Takahashi, "Cerebrovascular disease changes in somatosensory evoked potentials associated with unilateral lesions," Electroencephalogr Clin. Neurophysiol., vol. 35, pp. 463-473, 1973.

[9] H. Kato, Y. Sugawara, H. Ito, K. Onodera, C. Sato, and K. Kogure, "Somatosensory evoked potentials following stimulation of median and tibial nerves in patients with localized intracerebral hemorrhage: Correlations with clinical and CT findings," J. Neurological Sci., vol. 103, no. 2, pp. 172-178, Jun. 1991.

[10] M. A. W. Al-Rawi, F. B. Hamdan, and A. K. Abdul-Muttalib, "Somatosensory evoked potentials as a predictor for functional recovery of the upper limb in patients with stroke," J. Stroke Cerebrovascular Diseases: Official J. Nat. Stroke Assoc., vol. 18, no. 4, pp. 262-268, 2009.

[11] H. Feys, J. Van Hees, F. Bruyninckx, R. Mercelis, and W. De Weerdt, "Value of somatosensory and motor evoked potentials in predicting arm recovery after a stroke," J. Neurology, Neurosurgery, Psychiatry, vol. 68, no. 3, pp. 323-331, Mar. 2000.

[12] P. Tzvetanov and R. T. Rousseff, "Median SSEP changes in hemiplegic stroke: Long-term predictive values regarding ADL recovery," NeuroRehabilitation, vol. 18, no. 4, pp. 317-324, Jan. 2003.

[13] T. Allison, G. McCarthy, C. C. Wood, and S. J. Jones, "Potentials evoked in human and monkey cerebral cortex by stimulation of the median nerve," Brain, vol. 114, no. 6, pp. 2465-2503, 1991.

[14] A. Starr, B. Mckeon, N. Skuse, and D. Burke, "Cerebral potentials evoked by muscle stretch in man," Brain, vol. 104, pp. 149-166, 1981.

[15] G. Abbruzzese, A. Berardelli, J. C. Rothwell, B. L. Day, and C. D. Marsden, "Cerebral potentials and electromyographic responses evoked by stretch of wrist muscles in man," Exp. Brain Res., vol. 58, no. 3, pp. 544-551, 1985

[16] C. D. MacKinnon, M. C. Verrier, and W. G. Tatton, "Motor cortical potentials precede long-latency EMG activity evoked by imposed displacements of the human wrist," Exp. Brain Res., vol. 131, no. 4, pp. 477-490, Apr. 2000.

[17] E. Seiss, C. W. Hesse, S. Drane, R. Oostenveld, A. M. Wing, and P. Praamstra, "Proprioception-related evoked potentials: Origin and sensitivity to movement parameters," Neuroimage, vol. 17, no. 1, pp. 461-468, Sept. 2002.

[18] T. Mima, K. Terada, M. Maekawa, T. Nagamine, A. Ikeda, and H. Shibasaki, "Somatosensory evoked potentials following proprioceptive stimulation of finger in man," Exp. Brain Res., vol. 111, no. 2, pp. 233-245, Sept. 1996.

[19] J. W. van Strien, "Classificatie van links- en rechtshandige proefpersonen," Nederlands Tijdschrift Voor de Psychologie, vol. 47, pp. 88-92, 1992.

[20] F. Stolk-Hornsveld, J. L. Crow, E. P. Hendriks, R. van der Baan, and B. C. Harmeling-van der Wel, "The Erasmus MC modifications to the (revised) Nottingham sensory assessment: A reliable somatosensory assessment measure for patients with intracranial disorders," Clin. Rehabil., vol. 20, no. 2, pp. 160-172, Feb. 2006.

[21] A. R. Fugl-Meyer, L. Jääskö, I. Leyman, S. Olsson, and S. Steglind, "The post-stroke hemiplegic patient: 1. A method for evaluation of physical performance," Scand. J. Rehabil. Med., vol. 7, no. 1, pp. 13-31, 1975.

[22] R. Oostenveld and P. Praamstra, "The five percent electrode system for high-resolution EEG and ERP measurements," Clin. Neurophysiol., vol. 112, no. 4, pp. 713-719, Apr. 2001.

[23] S. F. Campfens, A. C. Schouten, M. J. A. M. van Putten, and H. van der Kooij, "Quantifying connectivity via efferent and afferent pathways in motor control using coherence measures and joint position perturbations," Exper. Brain Res. vol. 228, no. 2, pp. 141-153, May 2013 [Online]. Available: http://www.ncbi.nlm.nih.gov/pubmed/23665751

[24] R. Oostenveld, P. Fries, E. Maris, and J.-M. Schoffelen, "FieldTrip: Open source software for advanced analysis of MEG, EEG, and invasive electrophysiological data," Computational Intelligence Neurosci., vol. 2011, p. 156869, Jan. 2011.

[25] A. Fields, Discovering Statistics Using SPSS, 3rd ed. London, U.K.: SAGE Publications, 2009.

[26] R. W. Bohannon and M. B. Smith, "Interrater reliability of a modified Ashworth scale of muscle spasticity," Physical Therapy, vol. 67, no. 2, pp. 206-207, Feb. 1987.

[27] J. Schuurmans, E. de Vlugt, A. C. Schouten, C. G. M. Meskers, J. H. de Groot, and F. C. T. van der Helm, "The monosynaptic Ia afferent pathway can largely explain the stretch duration effect of the long latency M2 response," Exp. Brain Res., vol. 193, no. 4, pp. 491-500, 2009.
[28] L. Spieser, H. B. Meziane, and M. Bonnard, "Cortical mechanisms underlying stretch reflex adaptation to intention: A combined EEG-TMS study," Neuroimage vol. 52, no. 1, pp. 316-325, Aug. 2010 [Online]. Available: http://dx.doi.org/10.1016/j.neuroimage.2010.04.020

[29] J. A. Pruszynski and S. H. Scott, "Optimal feedback control and the long-latency stretch response," Exper. Brain Res., vol. 218, no. 3, pp. 341-359, May 2012.

[30] G. Abbruzzese, S. Ratto, E. Favale, and M. Abbruzzese, "Proprioceptive modulation of somatosensory evoked potentials during active or passive finger movements in man," J. Neurol. Neurosurg Psychiatry, vol. 44, no. 10, pp. 942-949, 1981.

[31] A. Starr and L. G. Cohen, "'Gating' of somatosensory evoked potentials begins before the onset of voluntary movement in man," Brain Res. vol. 348, no. 1, pp. 183-186, Nov. 1985 [Online]. Available: http:/ /www.ncbi.nlm.nih.gov/pubmed/4063823

[32] J. Kottner, L. Audigé, S. Brorson, A. Donner, B. J. Gajewski, A. R. Hróbjartsson, C. Roberts, M. Shoukri, and D. L. Streiner, "Guidelines for reporting reliability and agreement studies (GRRAS) were proposed," J. Clin. Epidemiol., vol. 64, no. 1, pp. 96-106, Jan. 2011.

[33] S. Prabhakaran, E. Zarahn, C. Riley, A. Speizer, J. Y. Chong, R. M. Lazar, R. S. Marshall, and J. W. Krakauer, "Inter-individual variability in the capacity for motor recovery after ischemic stroke," Neurorehabilitation Neural Repair, vol. 22, no. 1, pp. 64-71, 2008.

[34] G. Kwakkel, B. J. Kollen, J. van der Grond, and A. J. H. Prevo, "Probability of regaining dexterity in the flaccid upper limb: Impact of severity of paresis and time since onset in acute stroke," Stroke: J. Cerebral Circulation vol. 34, no. 9, pp. 2181-2186, Sep. 2003 [Online]. Available: http://www.ncbi.nlm.nih.gov/pubmed/12907818

[35] S. Graziadio, L. Tomasevic, G. Assenza, F. Tecchio, and J. A. Eyre, "The myth of the 'unaffected' side after unilateral stroke: Is reorganisation of the non-infarcted corticospinal system to re-establish balance the price for recovery?," Exper. Neurol., vol. 238, no. 2, pp. 168-175, Sep. 2012

[36] F. Tecchio, P. Pasqualetti, V. Pizzella, G. Romani, and P. M. Rossini, "Morphology of somatosensory evoked fields: Inter-hemispheric similarity as a parameter for physiological and pathological neural connectivity,” Neurosci. Lett., vol. 287, no. 3, pp. 203-206, Jun. 2000.

[37] T. Allison, A. L. Hume, C. C. Wood, and W. R. Goff, "Developmental and aging changes in somatosensory, auditory and visual evoked potentials," Electroencephalography Clin. Neurophysiol., vol. 58, no. 1, pp. 14-24, Jul. 1984.

[38] N. B. Lincoln, J. L. Crow, J. M. Jackson, G. R. Waters, S. A. Adams, and P. Hodgson, "The unreliability of sensory assessments," Clin. Rehabil., vol. 5, pp. 273-282, 1991.

[39] S. P. Dukelow, T. M. Herter, K. D. Moore, M. J. Demers, J. I. Glasgow, S. D. Bagg, K. E. Norman, and S. H. Scott, "Quantitative assessment of limb position sense following stroke," Neurorehabil. Neural Repair, vol. 24, no. 2, pp. 178-187, Feb. 2010.

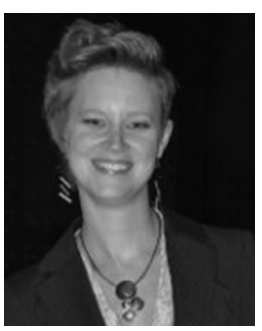

Sanne Floor Campfens received the M.Sc. degree in biomedical engineering with honors (cum laude) and the Ph.D. degree from the University of Twente, The Netherlands, in 2009 and 2014, respectively.

During her Ph.D. studies she developed methods to identify corticomuscular connectivity in motor control, both in normal subjects and subjects after stroke. She collaborated with the Leiden University Medical Center.

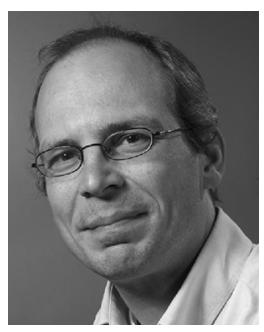

Carel G. M. Meskers received the M.D. and Ph.D. degrees.

$\mathrm{He}$ is a Consultant in neurorehabilitation and a Senior Researcher at VU University Medical Center, Amsterdam, the Netherlands. He holds a fellowship from the Dutch Brain Foundation ("Hersenstichting Nederland") to develop neurophysiological markers predicting recovery after stroke. He focuses on the application of basic control engineering paradigms, i.e., system identification and neuromechanics in daily clinical practice ultimo for goal directed therapy to enhance functional recovery in neurological diseases like stroke. 


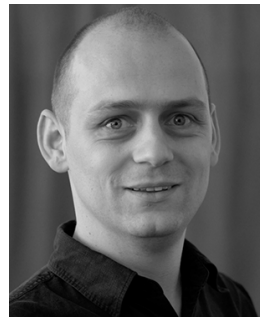

Alfred C. Schouten received the M.Sc. and Ph.D degrees in mechanical engineering from the Delft University of Technology, The Netherlands, in 1999 and 2004, respectively.

He holds a position as an Associate Professor at the Delft University of Technology and University of Twente. He is Cofounder of the Delft Laboratory for Neuromuscular Control. His research interest is the field of neuromuscular control and includes techniques to quantify the functional contribution of afnipulators, and system identification.

ferent feedback, neuromuscular modeling, haptic ma-

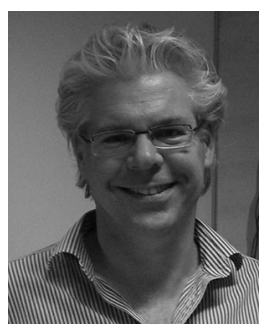

Michel J. A. M. van Putten received the M.D. degree from Leiden University and the Ph.D. degree in applied physics in 2000.

He heads the Department of Clinical Neurophysiology, Medisch Spectrum Twente, a large teaching hospital, and the Clinical Neurophysiology group at the University of Twente, The Netherlands. His main research interests include stroke, ischaemia and epilepsy, where EEG, TMS and modeling are used as important research tools.

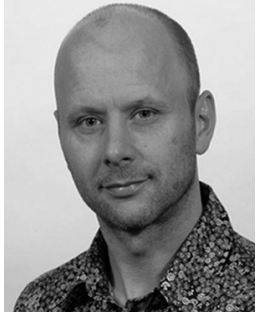

Herman van der Kooij received the Ph.D. degree with honors (cum laude) in 2000

$\mathrm{He}$ is currently a Professor in biomechatronics and rehabilitation technology at the Department of Biomechanical Engineering, University of Twente, The Netherlands, and Delft University of Technology, The Netherlands. His expertise and interests are in the field of human motor control, adaptation, and learning, rehabilitation robots, diagnostic, and assistive robotics, virtual reality, rehabilitation medicine, and neurocomputational modeling. $\mathrm{He}$ has published over 100 publications in the area of biomechatronics and human motor control. He is Associate Editor of IEEE TRANSACTIONS ON BIOMEDICAL ENGINEERING, member of IEEE EMBS technical committee of Biorobotics, and was member of several scientific program committees in the field of rehabilitation robotics, bio robotics, and assistive devices. 